\title{
Modifying Ways of Modern Local Furniture Design to The Ming-style Circle Chairs
}

\author{
Xue Ruizhu ${ }^{1}$, Zhao Hongsheng ${ }^{2}$ \\ ${ }^{1}$ Taiyuan University of technology, Taiyuan 030024, China \\ 2 Taiyuan University of technology, Taiyuan 030024, China
}

\begin{abstract}
In recent years, the scales and works for local Chinese original furniture designs have been rapidly increasing. This article elaborated the inner requirement for modern families to local furniture designs. And by comparing circle chairs of "Moreless", "Fnji", "pusu","Lost\&Found"and "LEXTO" with those made in Ming Dynasty, deeply analysis the modifying ways of modern local furniture design to the circle chairs with Ming style, such as tenon structure, matierals and so on. It aimed at increasing peoples' understanding for local furniture designs, improving peoples' attention for local original furniture design. Finally, this article supplied reference for the development of the local furniture design.
\end{abstract}

\section{Introduction}

Now more and more Chinese furniture designers escape the dominant ideology of Western design, using traditional heritage combined with the trend of Western ways instead, creating boutique furniture design. Ming-style furniture with its concise shape, well-structured, fine workmanship, decorated properly, simplified affordable, hard wood, texture and elegant temperament characteristics and vulgarity known to the world [1]. In the history of all kinds of furniture in the world, Only in China beautiful round-backed armchair can be found [2], it presents a unique oriental feeling, full of traditional cultural qualities of the Chinese nation. Foreign furniture design industry is projected to look for reference Ming-style chair and further development, such as Hans - Wagner's "Chinese chair" series, the Ming-style furniture, simplified strips, light and beautiful shape is formed [3]. Domestic independent design has made considerable progress in recent years, "Moreless", "Fnji", "pusu", "Lost\&Found"and "LEXTO" and other original brand in China to the Ming-style chair inspired design variety of modern chair, this paper analyze the shape, structure, pattern, materials, we hope these native design ideas generates inspiration for the future of furniture design.

\subsection{Contemporary local furniture design}

Contemporary furniture design is under local traditional aesthetic norms, applying modern materials and technology, showing a new interpretation of the classic essence of traditional Chinese culture, making furniture not only has an elegant, dignified Chinese flavor, but also has obvious modern features [4-5]. Contemporary designers through the transformation of traditional furniture, exhibits contemporary furniture design unique characteristics of the times, in which the expression of the Chinese-style, elegant and subtle, dignity, showing the Eastern spiritual pursuit, advocating a Chinese contemporary furniture design way "make the past serve the present".

\subsection{The evolution of Ming furniture}

Ming Dynasty furniture, especially from the Jiajing, Wanli Ming Dynasty to the Qing Dynasty Emperor Kangxi, Yongzheng (1522--1735 years), the products both from the quantitative point of view, or from the artistic value point of view is perfect, this period of two hundred years called traditional furniture Golden Age is well-deserved[6]. Ming-style have concise modeling, ergonomic structure of science, tenon joint structure, and on the aspect of materials selection and matching, attending to the wood itself natural texture and color, which bring the Ming-style furniture into a quiet and elegant literati aesthetic connotation. after a long history, Ming-style furniture increase more charm. 
Modern Transformation of the Ming-style furniture to meet the people's pursuit to peaceful and elegant mood and simple lifestyle. Soothing lines, smooth clear wood texture, artistic effect, meet the modern sense of belonging, a sense of belonging to find psychological symbols, to meet the people's need for furniture humanistic value. Local furniture and Scandinavian furniture, Japanese furniture in their own development process, resulting in a lot of thinking and the pursuit in common: respect for nature and the nice feeling from natural materials, pursue the harmony between man and nature; easy to use, avoiding affectation decoration, balance and moderation; humility, leisurely and elegant life attitude, so that the modernization of Ming furniture can better integrate modern home style, matching with modern furniture to create a favorable living environment.

\subsection{Contemporary domestic furniture design for the transformation of the Ming-style circle chair}

Ming-style circle chair have simple elements in general, on the seat pan is round wood, under the seat pan is square in cylindrical, simple rods in three direction, the back plate with a relief, a small angle between the rods and the goose neck, this can be regarded as a fundamental form of circle chair (refer with: Figure 1 Ming-style chair). Because of the elegant shape and unique, Ming-style circle chair has become a major reference for contemporary design.

Although the design of contemporary Native develop their own characteristics, but all vaguely embodies the spirit of Chinese ancient furniture. The following contrasts the "Moreless", "Fnji", "pusu", "Lost\&Found" and "LEXTO" with Ming-style circle chair, deeply analyze the transformation method of contemporary indigenous design on the Ming-style circle chair.

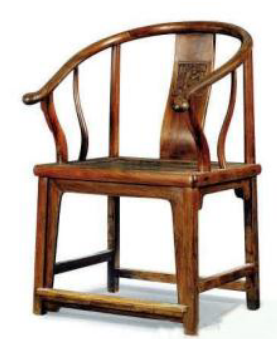

Ming-style chair

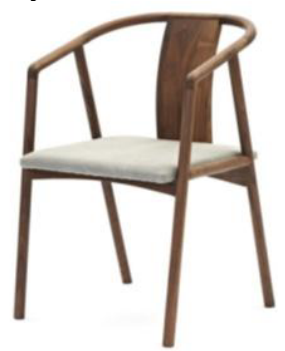

more or less- rare words chair more

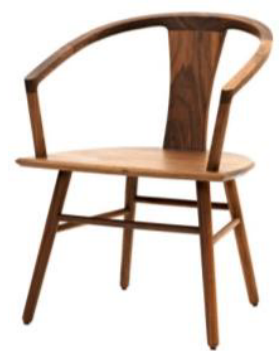

more or less-autumn chairs

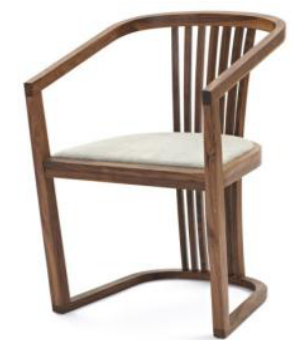

or less-together chair

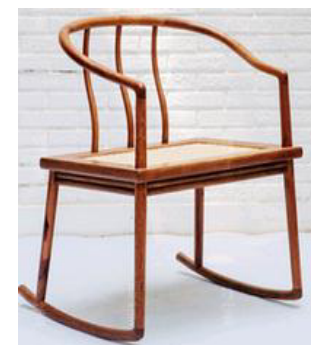

$\mathrm{Pu} \mathrm{Su}-Y$ unyao chair

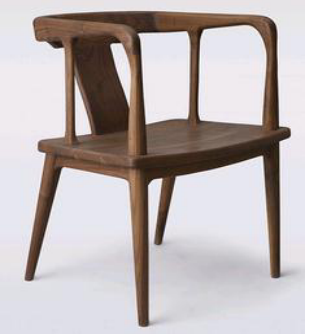

fnji-back chair

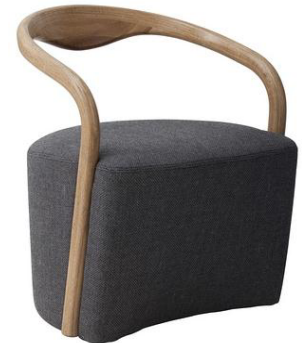

LEXTO -wood armchair

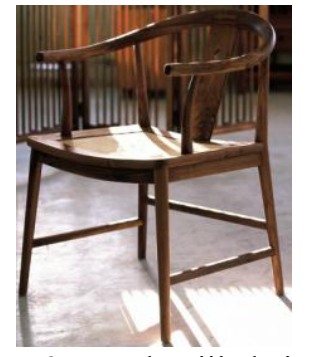

Lost\&Found -qiji chair
Figure 1. modern local circle chairs

\subsection{Simplified Shapes}

Different forms of modern circle chair, brought changes in use-pattern and mental function, meanwhile, as a map of the level of social development and people's social life. Modern circle chair no longer need to reflect this symbolic hierarchy, which makes the Ming-style chair from the solemn image increasingly developing in the direction of ease and rich flavor of life [7]. Contemporary local design reinforces the "concise shape "features of Ming Dynasty chair, so simplified design is the main method of modernize Ming-style circle chair(refer with Fig.1).

Most designs retain the coherent curve between brain and armrest, but transform it to more simple and smooth lines, or a little quadrate curve. Such as "more or less rare words chair " and " more or less-together chair ", using straight lines connected with arcs a dignified and generous character instead of the prime arc; and "LEXTO-wood armchair" use smooth rounded curves, reflecting the graceful and poetic.

There are three types to remould the brain and the back plate: the first type reserved ergonomic spine curves back plate, the second type is using the odd cylindrical root with the same curvature replaced the back plate, and the third type is simply remove backrest board, through widening the brain to support the body back instead;

All of designs removed the goose neck which is not actually supporting role, some also removed upside leg; on the transformation of seat pan, some reserve flat or transform to slightly curved surfaces, and others increase comfortable sit sense by adding cushions or rattan class material; on the transformation of foot doorpost and rods differentiated into two general renovation: remove the foot doorpost reserved rods or removed rods to increase the crossbar, while maintaining stable to make the leg more slender and light. especially " more or less-together chair " the lower part of the upper portion is almost symmetric, " 
LEXTO-wood armchair " transplant sofa form in the lower part , "Pu Su-Yunyao chair" increase in the bentwood chair bottom to become a rocking chair. The legs of "Fnji" and "Lost\&Found " are tapered, making it more lightweight.

Without exception, the transformation of formal ties with function together, based on the understanding of the scientific principles and the taste of potential users, to ensure the functional premise simplified the form, it retains unique characteristic curve of the brain and handrails, with simple flowing lines reflect the modern, light and overall slim, elegant and modern, carrying the culture, way of thinking and relationship with nature. It is worth mentioning that the progress and development of molding process combined with the designer's creativity, so chairs show a different shape and style." LEXTO-wood armchair" take wood armchair armrest and the back combined to form a complete structure, not only comfortable, but also has good toughness, smooth formal beauty.

On the handling of decorative patterns, decorative relief modeling traditional elements, although beautiful, but is not suitable for the needs of modern aesthetic taste, remove the decorative patterns can make furniture have much modern taste, and can save material, also avoid difficulties in cleaning.

\subsection{Simplifies the Tenon and Modern Technology Combine}

In order to improve production efficiency, adapt to mechanized production methods, appropriate to simplify the structure is imperative, which requires the relevant process improved. Tenon is major coupling member of traditional Ming-style circle chair, wherein the protruding part is called "sun", concave part is called "Mao" [8-9]. Traditional Ming Dynasty chair chairs circle combined with a dovetail, the back plate and the brain and seat pan connect in slot tenon, goose neck, together with the stick and armrests connected to the seat pan with a circular sleeve joints, legs connect seat pan with the length tonon.

Tenon joint structure in ancient times is a simple utility connections, but in modern it always as a decorative element to reflect the literati temperament and the traditional skills of furniture designers. More modern techniques can simplify the structure and reduce the difficulty of mechanization. Figure 1 is still a few cases of local modern armchair to analyze:

the tenon was made by machine instead of hand-made mechanical tenon. Dovetail, straight mortise and tenon and other simple internal structure can be mechanically forming. For example, the circle of "more or less- Autumn chair" use the three-mile circle straight tenon in connection, instead of the traditional wedge tenon, handrails and goose neck tenon connect by Yandai tenon, cylindrical thin teeth connect chair legs with a simple plug tenon; the circle of "Fnj- back chair " is square, so connect the brain and handrails by dovetail stitching, handrails and use dug Yandai goose neck tenon, sticks connect both sides insert with straight tenon, seat pan do not use the traditional grid corner tenon, using the three plate slot tenon splicing;
Ming-style chair modern multi-tenon joint structure combining hardware technology is. In ancient times make manufacture nails is difficult, but modern alloys such as copper, chrome and zinc alloy are more rigid and does not rust. In addition, non-hardwoods use tenon joint structure prone to cracking or loose change. For example, the seat pan and legs of " more or less-autumn chairs" connected each other by hardware, but is well hidden up;

useing bent wood technology, such as " LEXTO-wood armchair" use cantilevered armrests and backrest integrated design, modeling simple, use a wood bending technology, not with tenon.

\subsection{Combination of Wood and a Variety of Materials.}

Most of Ming and early Qing Period furniture was made of precious hard wood. Most of them have solid texture, elegant color, vivid and beautiful patterns [10]. Such as rosewood, Xichi wood, iron force, ebony and mahogany and so on. But these woods are not suitable for modern production.

Modern chair still using wood as the main material, the wood itself contains irreplaceable natural and simple emotions,is not only quite environmentally friendly materials if cut properly, but also play the role of moisture balance in the living room. Contemporary chairs of the local brand shown in Fig.1, where "More or Less", "Lost\&Found" transformation chair used black walnut, black walnut is a tough medium-density hardwood and diameter relatively uniform, can be used for large cross-section of the plate, the heartwood is light brown to dark brown, often with a crack purple and dark stripes, string section as large parabolic pattern, wood drying, low rigidity, good steam bending performance, ease of hand tools and machining, good fixing with nails, screws and glue, and strong corrosion resistance heartwood. "Pu Su-Yunyao chair" made of beech, yellow Burmese rosewood or birch. Where beech sturdy, even texture, good steam bending, texture clear and smooth, pastel colors, but in a dry environment easily deformed. Burmese rosewood wood densityis high, grain crisp and clear, with incense and hold nails performance is good, but the scarcity of expensive and difficult to dry and easy to moth-eaten. Birch is relatively heavy and hard, light brown to reddish-brown wood, easy processing, cut smooth, ring obviously, paints and glue good performance, but its moisture absorption, it is decayable, not wearable, and fiber shear force is poor .

In the use of materials can also be mixed and matched on a rattan palm, sponges and cloth and so on. Cloth makes people feel soft, warm, Figure 1 "More or Less -rare words Chair" and "together Chair" embedded in the surface of a piece of cloth chair cushion, not only enhance the comfort but also enhanced contemporary; " LEXTO -wood armchair " is use of whole cloth seat, a simple structure, to make the shape simple and elegant. The wood and rattan palm as a complex of two natural elements makes a sense of leisurely and affinity, such as the soft seat of "Pu Su-Yunyao chair", is all handmade rattan palm stretched. 


\section{Summary}

The local design has became an important part of Chinese culture, and will greatly promote the development and prosperity of China's manufacturing industry in the future . By summarizing the contemporary of local furniture design of the Ming-style circle chair transformation method to provide more local furniture design ideas. As China's domestic original brand, they have a natural Chinese culture gene, transformation of the shape of the chair reflects the commonality of simplification and slim, but performed in different ways; tenon traditional Chinese art, which had reservations, but reflect some thoughts and changes under the background of mechanization; in terms of materials, they have showed the insistence of wood materials and the absorb of modern furniture materials .

\section{References}

1. HUANG Wei, Zhang Meng, Wu Jian-feng Influences Of The Ming Literati'S Design On The Ming-Style Furniture Style Formation. Packaging Engineering, 2015, 10: 80-84.

2. CAO Yong Ming-style chair in perspective the essence of traditional Chinese culture. Forward Position, 2012, 22: $178-180$.
3. LI Wei-wei Innovative Design of Ming Style Furniture from the Perspetive of "Chinese Chair" by Hans Wegner . Packaging Engineering, 2015, 02: 95-98.

4. HU Tian-jun, WU Ya-wei Characteristics of Contemporary Furniture Design. Design Art (Shandong College of Arts and Crafts), 2010, 05: 11-12.

5. WANG Pei, Application Of Traditional Furniture Element In The Modern Furniture Design . Packaging Engineering, 2014, 24: 87-90.

6. LIAO Zhi-min Scholars and collectors Ming dynasty furniture. art market, 2013, 07: 54-58.

7. HUANG Mei-wei, XU Xue-min Study on The Evolution of The Modern Wenzhou Round-Backed Armchair By Analyzing Practical Case . Art and Design, 2013, 06: 74-75.

8. LI Min, WU Zhi-hui, MAO Yi-chao The Types of Mortise and Tenon In Modern Mahogany Furniture . Packaging Engineering, 2015, 04: 88-92.

9. WANG Di. Chinese Traditional Furniture Wood Structure and Technology Analysis Round-hacked Armchair . Northwest Forestry University, 2014, 05: 207-213.

10. LIU Shu-zhen, SUN Jian-ping Analysis on the Material Selection and Design of the Back of Ming-style Chairs. Forest Engineering, 2014, 02. 